\title{
Phytochemistry reviews: special issue on high altitude plants
}

\author{
Franz Bucar · Simon Gibbons
}

Published online: 25 July 2010

(C) Springer Science+Business Media B.V. 2010

The underlying theme of a the joint symposium organised by the Austrian Pharmaceutical Society and the Phytochemical Society of Europe was the highly specialised plants, lichens and fungi from distinct habitats at high altitudes. This symposium took place at the University of Innsbruck Centre in Obergurgl, Tyrol, Austria, April 26-29, 2009 at a time of year where there was still considerable snow in the village and on the upper slopes. The organisms that live in this harsh environment are influenced in their development by ecological factors including a short vegetation period, low temperatures and extensive temperature extremes, high UV-light impact, increased winds and of course considerable snow pressure. These factors lead to a high degree of biodiversity and a high diversity of unique secondary metabolites as a chemical response to these challenges. An international group of experts in the fields

The authors Franz Bucar and Simon Gibbons contributed equally.

F. Bucar $(\square)$

Institute of Pharmaceutical Sciences, University of Graz, Universitaetsplatz 4/1, 8010 Graz, Austria

e-mail: franz.bucar@uni-graz.at

S. Gibbons ( $\square)$

Department of Pharmaceutical and Biological Chemistry,

The School of Pharmacy, University of London, 29-39 Brunswick Square, London WC1N 1AX, UK e-mail: simon.gibbons@pharmacy.ac.uk of phytochemistry, biology and pharmacology met in Obergurgl to discuss "Plants from High AltitudePhytochemistry and Bioactivity" and this covered phytochemical, chemo-ecological, ethnomedicinal and pharmacological aspects as well as cultivating and breeding issues of high mountain plants and related organisms.

This special issue of Phytochemistry Reviews resulted from this symposium and presents recent work and provides views of this exciting research area from different angles which has rarely been covered before. Chemosystematic studies on Scorzonera, Arnica and Matricaria species (Asteraceae) provided highly interesting insights into the trends and causes of altitudinal variation of phenolic compounds. Due to its vertical spread, comparative studies on samples of Calluna vulgaris collected from wild populations at different altitudes were possible and investigations of markers of altitudinal variation among flavonoids and phenolic acids could be performed.

More than 40 species of Mallotus, a genus of high medicinal potential of the Euphorbiaceae family, are found in mountainous regions of Vietnam. An up-todate picture concerning their phytochemistry and biological properties has been drawn in the review included in this special issue.

In contrast to widely used Hypericum perforatum-St. Johns Wort, Hypericum species of the Paramos regions of Central and South America are rarely studied. In addition to an analysis of their phytochemistry, the viable and sustainable collection 
of plant material from ecologically sensitive habitats is also discussed.

The Andean flora provides an exiting variety of diterpenoids with antituberculous activity. Semisynthetic variation of the molecules of natural origin revealed some essential structural features for their biological action.

The final review highlighted a multidisciplinary approach in order to study the fundamental biological processes and the pharmaceutical and biotechnological potency of natural products. Modern pharmacognosy research as a link between chemistry, biology and medicine will continue to play a key role in this respect.
We believe that this area of natural product research deserves much wider attention and that studying biota at high altitude may give rise to unusual chemistry and distinct bioactivity. We want to thank the University of Innsbruck and in particular Prof Dr Hermann Stuppner for his pivotal role in organizing the conference that led up to the publication of this set of reviews and we thank Springer Verlag for publishing the conference proceedings in Phytochemistry Reviews. 